\title{
DIE ÜBERNAHME SYSTEMISCHER DENKWEISEN IN DER ÖFFENTLICHEN VERWALTUNG: EIN PARADIGMENWECHSEL
}

\section{REVIEW ARTICLE}

REBELO, Michele Alves Correa ${ }^{1}$

REBELO, Michele Alves Correa. Die Übernahme systemischer Denkweisen in der öffentlichen Verwaltung: ein Paradigmenwechsel. Revista Científica Multidisciplinar Núcleo do Conhecimento. 04-Jahr, Ed. 10, Band 01, S. 45-63. Oktober 2019. ISSN: 2448-0959,

Zugangslink: https://www.nucleodoconhecimento.com.br/business-administrationde/gedankensystemisch

\section{ZUSAMMENFASSUNG}

Angesichts des aktuellen politischen Szenarios In Brasiliens und der Veränderungen im Panorama der öffentlichen Verwaltung im 21. Jahrhundert zielt dieser Artikel darauf ab, die Reflexionen der Annahme des systemischen Denkens durch den öffentlichen Manager zu präsentieren, die den Einfluss des Meinungs dieses Gedankens entscheidungsfindung. Sie geht auf eine Forschung angewandter Natur, eines Sondierungsziels mit der Annahme bibliografischer Forschung zurück. Dies ist ein wichtiges Thema, da es dem öffentlichen Verwalter schwer fällt, Effizienz bei der Durchführung administrativer Tätigkeiten zu erreichen. Mit der Forschung konnte beobachtet werden, dass das systemische Denken des Managers die Richtung der öffentlichen Verwaltung beeinflusst, die Vorteile dieser Haltung können in den Dienstleistungen für die Gesellschaft und internen Handlungen der Institution sichtbar werden. Heute wird die Persönlichkeit in der Entscheidungsfindung beobachtet,

1 Master in Public Policy Management - UNIVALI. Facharzt für Straf- und Verfahrensrecht-Schule der Magistratse von Santa Catarina. Spezialist für öffentliches Sicherheitsmanagement - UNIVALI. Studium der Rechtswissenschaften - FEBE. 
aufgrund des politischen Einflusses des Staates und weil es ein sehr wettbewerbsorientiertes Szenario ist, in dem persönliche Eitelkeit und die Kultur der Unmittelbarkeit vorherrschen. Systemisches Denken trägt dazu bei, dass neue Visionen geteilt und kollaborativ werden. Der öffentlichen Verwaltung mangelt es an systematischer Innovation des Denkens, um sozialen Bedürfnissen gerecht zu werden und Effizienz zu erreichen.

Schlagworte: öffentliche Verwaltung, Public Manager, Effizienzprinzip, systemisches Denken.

\section{EINFÜHRUNG}

Die vorliegende Forschung schlägt vor, den Lesern die Reflexionen der Annahme systemischen Denkens durch den öffentlichen Manager aufzuzeigen und zu zeigen, wie dies das Leben der Menschen beeinflussen kann.

Seit dem 80. Jahrzehnt ist eine wachsende Bewegung auf der Suche nach modernen und effizienten Managementtechniken zu beobachten, die verbesserungen im öffentlichen Dienst bieten können (KETTL, 2005; PAULA, 2005; MANNING et al., 2009). Parallel zu dieser Realität stößt der Public Manager auf ein neues globales Umfeld, neue soziale Anforderungen, neue Technologien und Projektkonzepte.

Aufgrund dieser Faktoren hat sich die Art und Weise, wie öffentliche Dienstleistungen verwaltet werden, verändert. Das bürokratische Modell wird durch das Management ersetzt, und die politischen, sozialen und kulturellen Veränderungen beginnen, sich in die Denkweise der Gesellschaft einzumischen.

Trotz dieser Weiterentwicklung der Konzepte der Verwaltung und der öffentlichen Verwaltung wird es als die Schwierigkeit des öffentlichen Verwalters wahrgenommen, Effizienz bei der Durchführung seiner Tätigkeiten zu erreichen. Dies ist auf die Priorisierung der besonderen Interessen der Nominierten auf die Positionen der Leitung, Führung und Beratung sowie den politischen Einfluss auf die Entscheidungsfindung von öffentlichen Managern zurückzuführen. 
Nach Ansicht der Kammer (2009) impliziert die Funktion der Weisung, die durch den Zugang zu der als frei gestellt eingestuften öffentlichen Position ermöglicht wird, Erwartungen an die Wahrnehmung der Position, die mit den politischen Interessen derjenigen verbunden ist, die die Befugnis zur Ernennung haben. Aus diesem Grund ist persönliche Loyalität des Ernannten erforderlich. Dies ist eine prekäre Verbindung und kann jederzeit entlastet werden.

In der brasilianischen Verwaltungstradition hat nur der technischste Kern des Staates dazu, hohe Führungspositionen vor deutlich politischen Ernennungen zu bewahren (FLEURY, 2009). Im Idealfall würden diese Positionen von Personen besetzt, die über technischekenntnisse verfügen. Es wird jedoch in den meisten Fällen als subjektives Benennungskriterium angenommen.

Der Schwerpunkt dieser Arbeit liegt auf der Reflexion über eine neue Haltung des öffentlichen Managers angesichts dieses Problems. Es wird notwendig sein, Aspekte subjektiver Art, wie den Charakter des Managers, die das moralische oder soziale Verhalten und damit die Richtung eines guten oder schlechten Managements bestimmen, perfunctory zu behandeln.

Der öffentlichen Verwaltung fehlen neue Macht- und Führungspositionen. Eine systemische Innovation des Denkens sollte zu jedem Zeitpunkt in der Institution stattfinden. Es geht nicht nur darum, verschiedene Dinge zu tun, sondern die gleichen Dinge auf unterschiedliche Weise zu tun, wie Peter Drucker (2002) erwähnt, neue Zufriedenheitspotenziale zu schaffen.

Angesichts der Komplexität des Themas und einer systematischen Analyse haben wir das Recht und den Manager als offene Systeme, die sich in einer dynamischen Abstimmung beziehen. Sie befinden sich in einem kontinuierlichen und unaufhörlichen Prozess des Austauschs und des Austauschs mit der Umwelt. Organisationen sind Systemsysteme von Menschen (CHIAVENATO, 2000).

Unter der Vision und dem Beitrag der Sozialwissenschaften besteht die Möglichkeit, die Forschung auf der Grundlage von Bertalanfdas Theorie (1937) durchzuführen, die 
eine andere Sicht auf den wissenschaftlichen Reduktionismus hat, der bis dahin von der konventionellen Wissenschaft angewandt wurde.

Der systemische Ansatz ist laut Valena (2011) direkt mit der Lektüre der Komplexität von Argumentation, Beherrschung oder der Überreuigkeit von Emotionen bei menschlichen Entscheidungen und Anreizen zu einer effektiven Entscheidung verbunden.

Systematisch zu denken heißt, über Komplexität, Instabilität und Intersubjektivität nachzudenken. Ein Berufstätiger, der lebt - die Welt sieht und darin handelt - die Implikationen, die oben genannten Annahmen angenommen werden, kann als systemischer Berufstätiger betrachtet werden. Es muss den Blick des Beobachters haben - der Beobachter als Teil des Systems (VASCONCELOS, 2009).

Mit dem skizzierten Thema wird vorgeschlagen, die Reflexionen der Annahme von systemischem Denken und die Einflüsse, die diese Haltung im Leben der Menschen erzeugen kann, aufzuzeigen und die folgende Frage zu beantworten: Es wird möglich sein, Effizienz mit der Annahme von systemischem Denken durch Öffentlicher Manager?

Um das vorgeschlagene Ziel zu erreichen, wird die beschreibende Methode durch bibliographische Forschung verwendet.

Für Fonseca (2002) wird die bibliographische Forschung aus der Erhebung bereits analysierter theoretischer Referenzen durchgeführt und auf schriftlichen und elektronischen Mitteln wie Büchern, wissenschaftlichen Artikeln und Webseiten veröffentlicht.

Im nächsten Kapitel wird das Konzept der öffentlichen Verwaltung und, in einem bestimmten Punkt, der Manager vorgestellt werden. Das Prinzip der Effizienz wird dann perfunctory behandelt. Und schließlich werden wir das systemische Denken und die Reflexionen über diesen Look beim Aufbau eines neuen Paradigmas der öffentlichen Verwaltung vorstellen. 


\section{2. ÖFFENTLICHEVERWALTUNG}

In den letzten 30 Jahren wurde der Begriff "öffentliche Verwaltung" als Ersatz für die öffentliche Verwaltung verwendet und aus diesem Grund als polysemic Begriff betrachtet, da ein Teil der Doktrin versteht, dass die beiden Begriffe falsch sind. Andere argumentieren, dass das traditionelle Konzept gestört wurde, weil es ManagementTools der Geschäftswelt und damit ein breiteres Konzept umfasst.

Henri Fayol (1950), bereits im Jahrzehnt von 50, definierte Management als administrative Funktionen der Vorhersage, Organisation, Befehlierung, Koordination und Kontrolle.

Seit dem 80. Jahrzehnt haben die Regierungen mehrerer Länder Anstrengungen unternommen, um die öffentliche Verwaltung zu modernisieren, um die Reichweite der Regierung zu verringern und sie besser in der Verfolgung von Agilität funktionieren zu lassen. Die Bewertung der öffentlichen Verwaltung selbst ändert sich und wird dann durch Kriterien in der Nähe der von der privaten Verwaltung verwendeten abbalsamiert.

Als Ergebnis dieser Haltung entsteht das New Public Manegement (NPM), verbunden mit dem Kontext einer spezifischen Reform des Staates, dessen Modell gegen die konventionelle öffentliche Verwaltung ist, basierend auf dem Weberiane-VerletztenBürokratie-Modell besteht darin, eine Managementansatz, inspiriert von den Methoden der privaten Unternehmensführung, an die öffentliche Verwaltung (HOOD, 1995; HERNES, 2005), um eine bessere Reaktionsfähigkeit und bessere Leistung bei der Bereitstellung öffentlicher Dienstleistungen für die Bevölkerung zu gewährleisten (MANNING et al., 2009).

Perry und Kraemer (1983) sind der Ansicht, dass öffentliche Verwaltung eine Verschmelzung der normativen Ausrichtung der traditionellen öffentlichen Verwaltung und der instrumentalen Führung des Managements im allgemeinen Sinne ist. Mit anderen Worten, im ersten Aspekt umfasst die öffentliche Verwaltung Themen wie Demokratie und Rechenschaftspflicht sowie Werte wie Gerechtigkeit, Gleichheit und 
Wahrscheinlichkeit. Und in der zweiten, eine instrumentale Orientierung, mit der Idee, dass der öffentliche Sektor mit dem Privaten die Notwendigkeit teilt, seine Ziele wirtschaftlicher und effizienter zu erreichen.

Für Druker (1993) bezieht sich das Management nicht auf die Organisationshierarchie einer klassischen Verwaltung, sondern auf die Fähigkeit, systematische Wissensinnovationen zu fördern und daraus den maximalen Ertrag in der Anwendung auf die Produktion zu nehmen.

Im Gegensatz zur privaten Verwaltung muss sich die öffentliche Verwaltung von sozialen Werten leiten lassen. Es wird hier angenommen, dass management ein komplexer Akt ist, der uns der Welt der Politik näher bringt (BRUGU; SUBIRATS, 1996).

Wenn man über die historische Entwicklung der öffentlichen Verwaltung nachdenkt, bezieht man sich prospektiv auf eine Reflexion über die wahre Rolle des Staates heutzutage. Das Öffentliche zu verwalten, ist ein komplexer und herausfordernder Akt, da das Szenario sehr wettbewerbsfähig ist. Die persönliche Eitelkeit und die Kultur der Unmittelbarkeit herrschen in dieser Mitte. In jeder Legislaturperiode ändert sich die Dynamik der Verwaltung. Angesichts des politischen Einflusses, der das Land schwärmen, gibt es keine Kontinuität des Handelns aufgrund der Persönlichkeit in der Entscheidungsfindung.

Die Menschen sind erschrocken über die Nachricht von barbarischen Verbrechen, die in den Medien verbreitet werden. Sie sind sich jedoch nicht bewusst, dass eine überteuerte oder unvollendete Arbeit aufgrund von Misswirtschaft zu dem Chaos in der Bildung und Gesundheit einer bestimmten Region beigetragen haben könnte.

Cardoso Jr. (2001) argumentiert, dass die Verantwortung des Staates nicht nur darin besteht, die Dinge besser und effizienter zu machen. Darüber hinaus ist es die Aufgabe, die Bedingungen für die Transformation der wirtschaftlichen und sozialen Strukturen des Landes zu fördern, zu fördern oder sogar zu schaffen. Der Prozess beinhaltet die Neugestaltung der Struktur, der Verfahren und der Praktiken des 
öffentlichen Sektors, wobei Veränderungen von großem Ausmaß in die institutionelle Dimension einbezogen werden, die Fragen im Zusammenhang mit den Grundsätzen und Werten umfassen, die die Beziehung dieser Organisationen bestimmen. Mit gesellschaft und markt (FERLIE et al., 1999; Ormond LOFFLER, 1999; HERNES, 2005).

In diesem Sinne fehlt es der öffentlichen Verwaltung an systemischer Innovation, um sozialen Bedürfnissen gerecht zu werden und Effizienz zu erreichen. Die Übernahme von systemischem Denken durch den Manager kann dazu beitragen, dass neue Visionen zu gemeinsamen und kollaborativen Visionen werden. Die Vision des Ganzen ist für die Entscheidungsfindung von grundlegender Bedeutung.

Für Abrécio (2007) hat die öffentliche Verwaltung eine Reihe von Besonderheiten, die sich auf die Notwendigkeit beziehen, über Management- und demokratische Instrumente zu verfügen, um die Probleme zu bekämpfen, mit denen der Staat in der heutigen Welt konfrontiert ist. In diesem Zusammenhang sollten Formalismus und bürokratische Starrheit als Übel angegriffen werden, und die Meritokratie kann die Modernisierung fördern.

Gemeinsame Vision ist der erste Schritt, um Menschen, die einander nicht vertrauen, dazu zu bringen, zusammenzuarbeiten. Erstellt eine gemeinsame Identität. (SENGE,2009). Durch eine Kultur des Feedbacks tragen das Vertrauensverhältnis in das Team und die Anerkennung der Anstrengungen zur Effizienz des öffentlichen Managements bei, da es den Aspekt der Motivation erreicht.

Der Manager kann die im Laufe der Geschichte etablierten Konzepte (vor) transformieren. Obwohl Sie an das Prinzip der Legalität gebunden sind, können Sie etwas bewirken. Dazu muss sie eine neue Haltung einnehmen, den systemischen Look im Streben nach Effizienz, dessen Konzepte und Aspekte im Folgenden diskutiert werden. 


\section{1 ÖFFENTLICHER MANAGER}

Nach dem klassischen Konzept von Henri Fayol (1950) definiert sich der Manager durch seine Funktionen in der Organisation. Es ist die Person, die für die Interpretation der von der Institution vorgeschlagenen Ziele verantwortlich ist und durch Planung handelt, um die Ziele und Ziele zu erreichen.

Es kann dann gesagt werden, dass der Manager jemand ist, der den strategischen und operativen Plan entwickelt, die effektiven Mittel beurteilt, die Strukturen begreift und die am besten geeigneten politischen Regeln und Verfahren festlegt und letztlich die Durchführung von Projekten durch Die Mitte eines bestimmten Befehlstyps (oder einer bestimmten Führung).

Für Chiavenato (2004) als Manager oder Führer zu sein, ist es, eine globale Vision zu haben, eine Beziehung zwischen dem Menschen und seinem Arbeitsumfeld. Darüber hinaus lernt sie zu lehren und zu lernen, wobei letztere von entscheidender Bedeutung sind. Ausgehend von einer subjektiveren Prämisse stellt Peter Drucker (1993) fest, dass es durch den Charakter die Führung ausübt. In der gleichen Denklinie definiert Havard (2011) diese Tugend, die nicht nur ein intrinsischer Wert des Menschen ist, sondern eine dynamische Kraft, die die Handlungsfähigkeit erhöht, ein Merkmal, das für den Führer so notwendig ist. Tugend schafft Vertrauen, und ohne Vertrauen wird es unmöglich zu führen.

Ein öffentlicher Manager zu sein, ist eine Herausforderung, denn etwas Öffentliches zu verwalten, ist aufgrund der hart umkämpften politischen Landschaft ein komplexer Akt. Angesichts der häufigen Schlagzeilen in den Medien über Korruption, Abweichungen von öffentlichen Geldern, wurde ein Paradigma etabliert, dass öffentliche Dienstleistungen der Gesellschaft nicht effizient dienen.

In jeder Legislaturperiode ändert sich die Dynamik der Verwaltung, und es kommt zu radikalen Veränderungen. Dieser Prozess ist ein begrüßensimst, denn sonst gäbe es Diktatur oder Monarchie. Es ist jedoch eine Kontinuität der Aktionen und Projekte erforderlich. Es ist sehr wichtig, dass der Manager über die Wissenschaft und die 
Vorstellung verfügt, wie wichtig es ist, die Haltung bei der Ausführung alltäglicher Aufgaben zu ändern und wie diese Haltung das Leben der Menschen verändern kann. Eine kluge Entscheidung kann Bildung für bedürftige Kinder in einer bestimmten Region bedeuten. Mehr denn je schreit die Gesellschaft nach unterschiedlichen Ergebnissen. Es ist notwendig, den traditionellen Prozess des Denkens des öffentlichen Dienstes und der Umsetzung neuer Einstellungen zu überprüfen.

Der öffentliche Verwalter braucht ebenso wie bei der privaten Initiative die Eigenschaften und Eigenschaften, die es ihm ermöglichen, die vorgeschlagenen Änderungen zu fördern. Als führende Unternehmen im öffentlichen Sektor müssen die Führungskräfte mit Wissen, Fähigkeiten und Einstellungen ausgestattet werden, sowie mit einer starken Berufung, sich mit den verschiedenen Formen und Machtquellen zu befassen, die den öffentlichen Sektor durchdringen (MORGAN, 1996).

Die Annahme systemischen Denkens ist eng mit der Entwicklung der ethischen Tugenden des Managers und/oder Führers verbunden. Wir können einige Merkmale mit den systemischen Aspekten in Verbindung setzen, nämlich: Führung, die auf Zusammenarbeit und Einfluss auf geht, Kommunizität mit Schwerpunkt auf Beziehungen, Humanität mit Schwerpunkt auf dem Prozess, Netzwerkdenken, Flexibilität, Weitsicht mit Totalität und Dynamik (ANDRADE, 2016).

Die Verwaltung ist mit der Praxis des systemischen Denkens und der strategischen Hebelwirkung verbunden. Die Merkmale der Führung, im Kontext der menschlichen Interaktion, die die Prozesse der Optimierung des Lernens begünstigt, sind auf die Ausübung der Teilnahme auf allen Ebenen und für den Aufbau von Teams ausgerichtet (ZANELLI,2008).

Die Idee der Unwirksamkeit der öffentlichen Dienstleistungen sollte aufgegeben und der politische Einfluss verringert werden. Es ist notwendig zu verstehen, dass, wenn die Aufmerksamkeit auf einen isolierten Teil konzentriert wird, der auf eingeschränkte Interessen ausgerichtet ist, es nicht möglich sein wird, das ganze System wahrzunehmen, da es von grundlegender Bedeutung ist, über das Ganze 
nachzudenken und nicht in den Phasen, wenn man Richtungen korrigieren und Effizienz erreichen will, dessen thematische wird im folgenden Thema angezeigt.

\section{PRINZIP DER EFFIZIENZ}

Zunächst muss festgestellt werden, dass es sich bei den Verwaltungsgrundsätzen um grundlegende Leitlinien handelt, die die Arbeitsweise öffentlicher Stellen untermauern und unterstützen, wenn die mit der öffentlichen Verwaltung verbundenen Zuschreibungen wirksam sind.

Das in der Kunst vorgesehene Prinzip der Effizienz. 37 des CF von 1988, hatte seinen Ursprung im Privatrecht, in die öffentliche Verwaltung eingefügt, in der Verwaltungsreform.

Effizienz im öffentlichen Sektor ist eine Voraussetzung für den neuen globalen Trend, um kollektiven Interessen schnell und mit effektiven Ergebnissen gerecht zu werden. Sie sucht einen Staat, der seine Funktionen effektiv plant, entwickelt und ausführt, indem er neue Techniken und Gewohnheiten nutzt, die zufriedenstellende Ergebnisse erzielen.

Das Prinzip der Effizienz kann in zwei Verzerrungen verstanden werden: erstens in Bezug auf die Wirkungsweise des öffentlichen Agenten, von dem die bestmögliche Leistung seiner Zuschreibungen erwartet wird, die auf die besten Ergebnisse abzielt, und zweitens in Bezug auf die Art und Weise der Organisation, Struktur und Disziplin der öffentlichen Verwaltung, auch mit dem Ziel, die besten Ergebnisse bei der Erbringung öffentlicher Dienstleistungen zu erzielen (DI PIETRO, 2014).

Die Verwaltungstätigkeit muss von Führungskräften durchgeführt werden, die sich mit der Wirksamkeit der Erbringung öffentlicher Dienstleistungen befassen. Das Hauptmerkmal des Managers sollte systemisches Denken sein, um den gesamten Prozess zu visualisieren. Effizientes Management hat die soziale Entwicklung als Ihren Strang. Daher sollte das Hauptziel des Managers in der Verwendung der besten Mittel zur Befriedigung der kollektiven Bedürfnisse stehen. 
Der Wechsel der Haltung hängt mit der neuen Vision von Managern und Bürgern in Bezug auf die Verwaltung der öffentlichen Sache zusammen. Es ist notwendig, sich von der überholten Ansicht zu trennen, dass sich das Interesse des Staates mit dem privaten Interesse überschneidet.

Der moderne Konstitutionalismus positionierte den Menschen nicht nur im Epizentrum der Rechtsordnung und garantierte einen differenzierten Schutz der individuellen Grundrechte, sondern auch nachgebildete Interessen kollektiver Natur, die den individuellen Bereich übersteigen. Zweck der Förderung des Genusses von Rechten durch alle Mitglieder der politischen Gemeinschaft (BINENBOJM,2010).

Der Begriff des öffentlichen Interesses ist als die maximale Verwirklichung aller individuellen und kollektiven, rechtlich geschützten Interessen zu interpretieren. In der gegenwärtigen demokratischen Rechtslage überschneiden sich die Grundrechte der Gesellschaft, und ihre Befriedigung muss von dem Staat verfolgt werden, der über diesem anderen Interesse steht.

Die mangelnde Kontrolle der Gesellschaft über den Staat, der nach wie vor von besonderen Interessen dominiert wird, wird als negativer Faktor in der öffentlichen Verwaltung genannt. Für den Sohn (2005) stellt die Bewahrung des privaten Bereichs des Einzelnen in Verbindung mit der Förderung der Wünsche der politischen Gemeinschaft das wirkliche öffentliche Interesse dar, das von der Verwaltung verfolgt wird.

Damit sich die Effizienz erheblich verbessern kann, ist nicht nur die Demokratisierung des politischen Systems notwendig, sondern auch die Einbeziehung interner demokratischer Mechanismen in die öffentliche Verwaltung, die mit der Bildung öffentlicher Politiken und der Machtteilung bei der Entscheidungsfindung.

Öffentliche Manager, Bürger und andere Akteure sind Teil eines dynamischen, offenen und interaktiven Governance-Systems, in dem die Autorität des Staates auf unterschiedliche Weise genutzt wird, um verschiedene öffentliche Ergebnisse zu erzielen. 
In diesem Zusammenhang hat die Teilhabe der Gesellschaft einen unermesslichen Wert, da sie eine aktive Bürgerschaft fördert. Die Stärkung der Gemeinschaften und der Bürgergeist können einen Unterschied in der öffentlichen Verwaltung und damit in den Ergebnissen der staatlichen öffentlichen Dienstleistungen bewirken.

Auf der anderen Seite sollte der öffentliche Manager sein Handeln unter der Ägide des systemischen Denkens widerspiegeln, dessen Thema im Folgenden diskutiert wird.

\section{SYSTEMISCHES DENKEN}

Durch den Akt des Denkens modellieren die Menschen die Welt und interagieren nach ihren Wünschen, Plänen und Zielen. Die Konzepte und Prozesse der Kognition, Seton, Bewusstsein und Imagination (ANDRADE, 2016) können mit Denken in Verbindung gebracht werden. Mit einem Fokus auf die Evolution der Wissenschaft können wir die vorherrschenden kartesischen, komplexen und systemischen Gedanken zitieren.

Der systemische Gedanke entstand im TWENTIETH-Jahrhundert, im Gegensatz zu dem reduktionistisch-mechanistischen Denken von Descartes und Newton, das auf dem traditionellen Wissenschaftsparadigma von Vasconcellos (2009) basiert, dessen Grundannahmen in Einfachheit, Stabilität und Objektivität.

Die Annahme der Einfachheit beruht auf der Überzeugung, dass es notwendig ist, die Parteien zu trennen, um das Ganze zu verstehen. In diesem Look interpretiert Cunha (2006), dass die Reduktion eine weitere Operation auf der Suche nach Einfachheit ist, die das Vielfältige vereint. Indem er ein komplexes Phänomen findet, sucht der Wissenschaftler seine Reduktion auf ein anderes einfacheres und bereits gut verstandenes Phänomen.

Die Annahme der Stabilität liegt in dem Glauben, dass es Stabilität in der Welt gibt und es regelmäßig Wiederholungen gibt. Unter der Annahme der Objektivität stellt sie den Glauben dar, dass es möglich ist, die Welt, wie sie in wirklichkeit zusammengesetzt ist, unter Berücksichtigung des wesentlichen Kriteriums des wissenschaftlichen Denkens zu kennen (CUNHA, 2006). 
Das Paradigma der traditionellen Wissenschaft entwickelte und etablierte sich durch linear-kartesisches Denken in den physikalischen und exakten Wissenschaften und wurde zu einem Modell der Wissenschaft. Die Physiker handelten auf der Grundlage der Annahmen von Einfachheit, Stabilität und Objektivität und erklärten die physische Welt und entwickelten ausgeklügelte Technologien, die die Beziehungen des Menschen zur Natur zunehmend veränderten (VASCONCELLOS, 2009).

Mit der Überzeugung, dass es einen Unterschied zwischen Mensch und Natur gibt und dass menschliche Phänomene subjektiver Natur sind, ist der Bruch zwischen Naturwissenschaften (objektiv) und Humanwissenschaften etabliert. Nach Vasconcellos (2009) übernahmen die Physikwissenschaften leicht die drei erkenntnistheoretischen Annahmen. Die biologischen Wissenschaften übernahmen die Annahme der Objektivität und hatten Probleme mit denen der Einfachheit und Stabilität, während die Geisteswissenschaften Schwierigkeiten in Bezug auf die drei Annahmen hatten, insbesondere die der Objektivität.

Das systemische Denken schlägt dagegen die Paradigmen von Komplexität, Instabilität und Intersubjektivität vor, die sich unglaublich in die analytische Psychologie von Carl Gustav Jung integrieren (VASCONCELLOS, 2009).

Ein Profi, der lebt, die Welt sieht und in ihr die Implikationen handelt, die sich aus der Annahme von Komplexität, Instabilität und Intersubjektivität für sich selbst nehmen, kann als systemischer Berufstätiger betrachtet werden (VASCONCELLOS, 2009).

Das systemische Paradigma, das die Annahmen von Komplexität, Subjektivität, Instabilität betrachtet, hat als Prinzipien die Flexibilität und Plastizität, die sich in den Systemen durch Auto-Organizativas Eigenschaften manifestieren, die aus dem Chaos, Übernimmt Aspekte der Selbsterneuerung und der automatischen Umsetzung. So können offene Systeme, die weit vom Gleichgewicht entfernt sind, erhalten, reproduzieren, erneuern, sich anpassen und sich weiterentwickeln. So sind die Anpassungsprozesse auf der einen und der anderen Seite offensichtlich, die des Lernens, der Entwicklung und der Evolution (COSTA, 2007). 
Cunha (2006) drückt die Vorstellung aus, dass systemisches Denken Denken in Bezug auf Zusammenhänge, Beziehungen, Kontext, Wechselwirkungen der Elemente eines Ganzen bedeutet; Um die Dinge in Bezug auf Netzwerke, Webs und Communities zu sehen.

Systemisches Denken leugnet die wissenschaftliche Rationalität nicht, sondern glaubt, dass es keine ausreichenden Parameter für die menschliche Entwicklung und für die Beschreibung des materiellen Universums liefert. Aus diesem Grund versucht sie, in die entgegengesetzte Richtung des Reduktionismus zu blicken und dem Ganzen mehr Gewicht zu verleihen als auseinander.

Es setzt eine Methodik der Denkprozesse voraus, die die Existenz des Ganzen konfigurieren. Das heißt, es ist notwendig, die Systeme global zu studieren, um alle ihre Interdependenzen einzubeziehen, weil jedes der Elemente, wenn es zu einer größeren Funktionseinheit zusammengesetzt wird, Qualitäten entwickelt, die nicht in ihren Komponenten enthalten sind. Isolates (BERTALANFFY, 2009).

Capra (1996) argumentiert, dass fragmentiertes Denken nicht in der Lage ist, die Vernetzung globaler Probleme anzugehen, sowohl auf den höheren Ebenen der Gesellschaft als auch auf der individuellen Ebene. In diesem Sinne liegt das Wesen des systemischen Denkens in der Mentalitätsänderung, was bedeutet, Zusammenhänge statt von linearen Ketten zu sehen und Veränderungsprozesse statt Momentaufnahmen zu sehen (Senge, 2009). In dieser Sammlung sollten Änderungen nicht als isolierte Aktionen betrachtet werden, sondern als eine Reihe voneinander abhängiger Maßnahmen, die Teil der Planung von Verbesserungen der Organisation sind.

Es ist eine Möglichkeit, das Wissen über die Welt zu analysieren und zu denken, durch Systeme. Eine systemische Sicht oder "systemisches Denken" bedeutet, im Auge zu behalten, was man lösen will, die richtige oder die am besten geeignete Art und Weise zu wählen. Man kann sagen, dass dies eine Fähigkeit ist, die eine Person erwirbt, um 
die Ereignisse und ihre möglichen Folgen zu analysieren, mit dem Ziel, eine einzigartige Lösung zu schaffen, die die Erwartungen aller Beteiligten betrachtet.

Sowohl in der privaten als auch in der öffentlichen Verwaltung gibt es keine Einheitlichkeit bei der Annahme systemischer Denkweisen durch Manager. Die meisten öffentlichen Agenten kennen das Konzept nicht einmal. Die Bedeutung des systemischen Denkens im Management kann durch Zanellis Worte bekräftigt werden:

Die Verwaltung ist mit der Praxis des systemischen Denkens und der strategischen Hebelwirkung verbunden. Die Merkmale der Führung, im Kontext der menschlichen Interaktion, die die Prozesse der Optimierung des Lernens privilegiert, sind auf die Ausübung der Teilnahme auf allen Ebenen und für den Aufbau von Teams ausgerichtet (ZANELLI,2008, S. 13).

Im Jahr 2008 führte die National Quality Foundation (FNQ), die in den renommiertesten Spitzenmodellen der Welt vertreten ist, eine virtuelle Umfrage mit 196 brasilianischen Führungskräften durch. Die Ergebnisse zeigten, dass die Mehrheit der Befragten das Konzept des systemischen Denkens nur teilweise kannte.

Die große Sorge der systemischen Wissenschaftler ist die Erkenntnis, dass die Notwendigkeit eines tiefgreifenden Wandels der Wahrnehmung und des Denkens, um das Überleben zu sichern, noch nicht die Mehrheit der Manager erreicht hat, noch die Verwalter und Lehrer der Unsere wichtigsten Universitäten (CAPRA, 1996).

Wenn Menschen die Welt anders wahrnehmen, entstehen neue Überzeugungen und Annahmen, die die Entwicklung neuer Fähigkeiten und Fähigkeiten ermöglichen und einen kontinuierlichen Zyklus des organisatorischen Lernens bilden.

Peter Senge (2009) stellt fest, dass systemisches Denken als Disziplin dargestellt werden kann, eine Studie mit Theorie und Praxis, die es ermöglicht, die Vision der Montage zu entwickeln, eine Struktur, um die Zusammenhänge anstelle der Dinge zu sehen, Um Veränderungsmuster zu sehen Anstelle von statischen 'Snapshots '. Der Manager, wie er systemisches Denken annimmt, kann einen Unterschied in der öffentlichen Verwaltung machen und die Realität des Landes verändern. Mit diesem 
neuen Look können Sie die Handlungen der Institution durchführen, Richtlinien und Verfahren interpretieren und die Prozesse und internen Praktiken an die gesetzlich festgelegten Anforderungen anpassen.

Es braucht Innovationen im öffentlichen Management. Der Manager muss die Rolle übernehmen und hört auf, als bloße Randaliere zu agieren, die Aufgaben mutig zu erfüllen. Systematisch um der sozialen Wohlfahrt willen zu denken.

Es ist ein komplexes Thema, weil die Änderung der Haltung des Managers, bei der Annahme systemischen Denkens in der Ausübung des Managements, mit subjektiven Kriterien wie Charakter zusammenhängt, deren Tugend nicht gemessen oder bewertet wird, wenn die Eingabe dieser Beruf in der öffentlichen Tätigkeit, unabhängig davon, ob es sich um eine öffentliche Ausschreibung oder eine Nominierung durch Nominierung handelte.

Wie in der Forschung dargelegt, nimmt die öffentliche Verwaltung jedoch zu, wodurch die politische Kapazität des Staates erweitert wird, um "die Politik gut zu machen", um der Gesellschaft besser zu dienen und damit Effizienz zu erreichen. Immer mehr Autoren befürworten die theoretische Aufgabe des Prinzips der Vorherrschaft. Öffentliche Interessen und private Interessen sind eindeutig nicht antagonistisch, sondern komplementär (JUSTEN FILHO, 2005).

Die Forschung ließ ableiten, dass die Anwendung der Konzepte des systemischen Denkens einen wesentlichen Mehrwert für verschiedene Forschungsbereiche und in Organisationenbereichen schaffen kann. In diesem Sinne kann die Anwendung des systemischen Ansatzes in anderen Bereichen als relevanter akademischer Beitrag entlarvt werden.

Es ist daher berechtigt, zu bestätigen, dass das Ziel dieser Studie erreicht wurde, und es ist möglich zu bestätigen, dass die Annahme des systemischen Denkens durch den Verwalter in direktem Zusammenhang mit dem Grundsatz der Effizienz steht. 
Für zukünftige Studien wird vorgeschlagen, Forschungen in öffentlichen Verwaltungsorganen durchzuführen, mit dem Ziel, die systemischen Verhaltensmerkmale von Managern zu überprüfen und Praktiken für das systemische Management mit einem Schwerpunkt auf dem Verhalten der Menschen vorzuschlagen. .

\section{ABSCHLIEßENDE ÜBERLEGUNGEN}

Nach der Analyse der Dokumente in der Literatur gefunden, war es möglich, festzustellen, dass die Annahme von systemischem Denken durch den Manager kann die Richtung der öffentlichen Verwaltung des Landes beeinflussen. Dies ist eine Fähigkeit, die eine Person erwirbt, um die Ereignisse und ihre möglichen Folgen zu analysieren, mit dem Ziel, eine einzigartige Lösung zu schaffen, die die Erwartungen aller Beteiligten betrachtet. Die Vorteile dieser neuen Vision/Haltung können in den Dienstleistungen der Gesellschaft und den internen Handlungen der Institution sichtbar sein, da sie in direktem Zusammenhang mit der Entscheidungsfindung steht.

Zu diesem Zweck braucht der öffentliche Verwalter ebenso wie in privater Initiative die Eigenschaften und Eigenschaften, die es inm ermöglichen, die vorgeschlagenen Änderungen zu fördern. Als Führer des öffentlichen Sektors müssen die Führungskräfte mit Wissen, Fähigkeiten und Einstellungen ausgestattet werden, sowie mit einer starken Berufung, sich mit den verschiedenen Formen und Machtquellen zu befassen, die den öffentlichen Sektor durchdringen (MORGAN, 1996).

Die Übernahme von systemischem Denken ist eng mit der Entwicklung der ethischen Tugenden des Managers verbunden. Das Weltbild ist individuell. Bald gibt es keine Möglichkeit, eine Einheit oder Kontrolle in der Art und Weise des Denkens und Handelns zu haben. In dieser Zeitzeit sollte es nicht als isolierte Aktionen betrachtet werden, sondern als eine Reihe unabhängiger Maßnahmen, die Teil der Planung von Verbesserungen der Organisation sind. 
Systemisches Denken "hilft zu verstehen, wie man Systeme effektiver verändert und wie man besser mit den Prozessen der natürlichen und sozialen Welt in Einklang steht" (VALEN-A, 2011).

Die Organisationen, die in Zukunft wirklich erfolgreich sein werden, werden diejenigen sein, die entdecken, wie man in den Menschen das Engagement und die Fähigkeit, auf allen Ebenen der Organisation zu lernen, kultiviert werden kann (SENGE,2009).

Daher sollte berücksichtigt werden, dass die vorliegende Studie beabsichtigt, eine noch beginnende Perspektive auf ein strategisches Thema für öffentliche Stellen in Bezug auf das Management zu diskutieren, ohne die Absicht zu haben, das Thema auszuschöpfen.

\section{REFERENZEN}

ABRUCIO, Fernando Luiz. Trajetória recente da gestão pública brasileira: um balanço crítico e a renovação da agenda de reformas. Edição Especial Comemorativa. Rio de Janeiro: Revista de Administração Pública (RAP), 2007.

ALVAREZ, Maria Esmeralda Ballestero. Organização, sistemas e métodos. São Paulo: McGraw-Hill, 1990.

ALVES, João Bosco da Mota. Teoria Geral de Sistemas - Em busca da interdisciplinaridade. Florianópolis. Instituto Stela, 2012.

AMARAL, Antônio Carlos Cintra do. O princípio da eficiência no direito administrativo. Revista Diálogo Jurídico, Salvador, CAJ - Centro de Atualização Jurídica, n.14, jun./ago. 2002.

ANDRADE, A; Rodrigues, L; Seleme A; Souto, R; Pensamento sistêmico: Caderno de campo. $O$ desafio da mudança sustentada nas organizações e na sociedade. Porto Alegre: Bookman, 2006. 
BINENBOJM, Gustavo. Da Supremacia do Interesse Público ao Dever de Proporcionalidade: Um novo paradigma para o Direito Administrativo. In: SARMENTO, Daniel (org.). Interesses públicos versus Interesses privados: desconstruindo o princípio da supremacia do interesse público. Rio de Janeiro: Lumen Juris, 2010.

BERTALANFFY, L. von. Teoria Geral dos Sistemas: Fundamentos, desenvolvimento e aplicações. São Paulo: Editora Fundação Perseu Abramo, 2006.

BRASIL. Constituição da republica Federativa do Brasil: 1988. 25. Ed. Brasília: Câmara dos Deputados, Coordenação de Publicações, 2012.

CÂMARA, Leonor Moreira. Fatores do Pensamento Sistêmico como Potencializadores de Sucesso de Projetos de Software no Setor Público.organização da administração pública Federal Brasileira: uma introdução ao estudo da organização da direção pública na perspectiva de estudos organizacionais. Rio de Janeiro: Revista de Administração Pública (RAP), 43(3), maio/junho 2009.

CAPRA, F. O Ponto de Mutação. A ciência, a sociedade e a cultura emergente. Trad. Álvaro Cabral, São Paulo: Cultrix, 2006.

CAPRA, Fritjof. A Teia da Vida. Uma Compreensão Cientifica dos Sistemas Vivos. São Paulo (SP). Editora Cultrix. 1997.

CARNEIRO, R., MENICUCCI, TMG. Gestão pública no século XXI: as reformas pendentes. In Fundação Oswaldo Cruz. A saúde no Brasil em 2030 - prospecção estratégica do sistema de saúde brasileiro: desenvolvimento, Estado e políticas de saúde [online]. Rio de Janeiro: Fiocruz//pea/Ministério da Saúde/Secretaria de Assuntos Estratégicos da Presidência da República, 2013. Vol. 1. pp. 135-194. ISBN 978-85-8110-015-9. AvailablefromSciELO Books.

CHIAVENATO, Idalberto. Recursos Humanos. O Capital Humano das Oganizações. SãoPaulo:Atlas, 2004. 
CHIAVENATO, Idalberto. Iniciação à Organizações e Controle.São Paulo: McGrawHill, 1989.

COSTA, WedjaJosefa Granja. Socionomia de base sistêmica: Método de apoio à gestão de grupos na organização. Dissertação de Mestrado. Fortaleza: Universidade Federal do Ceará, 2007.

CUNHA, Aura Celeste Santana. Pensamento Sistêmico e tecnologia educacional: a metodologia WEBQUEST. Dissertação de Mestrado Profissional em Computação. Fortaleza: Universidade Estadual do Ceará, 2006.

DRUCKER, Peter. The effective executive.Harper Collins Publishers, 1993.

DRUCKER, Peter F. Administrando para o Futuro: os anos 90 e a virada do século. Tradução de Nivaldo Montigelli Jr. São Paulo: Pioneira Thomson Learning, 2002.

FALCONI, Vicente. O verdadeiro Poder.Nova Lima: INDG Tecnologia e Serviços Ltda. 2009.

FAYOL, Henri. Administração Industrial e Geral.São Paulo. Atlas, 1990.

FLEURY, S. J. F. Redes de proteção: incentivos, escolhas e comportamentos na política brasileira. 2009. Tese (Doutorado) - Faculdade de Filosofia e Ciências Humanas da Universidade Federal de Minas Gerais, Belo Horizonte, 2009.

HERNES, T. Four ideal-types organizational responses to the new public management reforms and some consequences. International Review of Administrative Sciences, v. 71, n. 1, p. 5-17, 2005.

HOOD, C. A public management for all seasons? Public Administration, . 69, $\mathrm{n}$. $1,1991$. 
JUSTEN FILHO, Marçal. Curso de Direito Administrativo. São Paulo: Saraiva, 2005.

GOMES, Lauren Beltrão et al . As origens do pensamento sistêmico: das partes para o todo. Pensando familiar, Porto Alegre, 2014.

KOLTER, John P. Liderando Mudanças. Rio de Janeiro: Elsevier, 2013.

LAKATOS, Eva Maria; MARCONI, Mariana de Andrade. Fundamentos de Metodologia Científica. 4 ed. São Paulo: Atlas, 2001.

LIMA, Joilson Souza de. O Planejamento Estratégico como Ferramenta de Gestão. Revista Científica Multidisciplinar Núcleo do Conhecimento. Ano 03, Ed. 03, Vol. 03, pp. 58-69, Março de 2018. ISSN:2448-0959.

MEIRELLES, Hely Lopes. Direito Administrativo Brasileiro. São Paulo: Malheiros, 2002.

MANNING, N. et al. Reformas de gestão pública: o que a América Latina tem a aprender com a OCDE.In: MEDEIROS, P. C.; LEVY, E. (Orgs.). Novos caminhos da gestão pública: olhares e dilemas. Rio de Janeiro: Qualitymark; Brasília: CONSAD, 2009. p. 97-148.

MORAES, Germana de Oliveira. Controle Jurisdicional da Administração Pública. São Paulo: Dialética, 1999, p. 127.

MORGAN, Goreth. Imagens da Organização. São Paulo: Atlas, 1996.

PIETRO, Maria Sylvia Zanella Di. Direito Administrativo. São Paulo: Atlas, 2002.

SENGE, Peter M. A quinta disciplina: arte e prática da organização que aprende. $25^{\text {a }}$ ed. Rio de Janeiro: BestSeller, 2009.

SEMLER, Ricardo. Virando a própria Mesa: Uma história de Sucesso Empresarial. Rio de Janeiro, 2002. 
VALENÇA, Antônio Carlos. Aprendizagem Organizacional: 123 aplicações práticas de arqueótipos sistêmicos. São Paulo:SENAC, 2011.

VASCONCELLOS, Maria José Esteves. Pensamento Sistêmico: O novo paradigma da ciência. 9a ed. Campinas-SP: Papirus,2010.

ZANELLI, José Carlos. Interação Humana e Gestão: a construção psicossocial das organizações de trabalho. São Paulo: Casa do Psicólogo, 2008.

Eingereicht: Oktober 2019.

Genehmigt: Oktober 2019. 